\title{
Prevalence and molecular characterization of methicillin-resistant Staphylococcus aureus with mupirocin, fusidic acid and/or retapamulin resistance
}

Wenjing Chen ${ }^{1 \dagger}$, Chunyan $\mathrm{He}^{1+}{ }^{1}$, Han Yang ${ }^{1}$, Wen Shu ${ }^{1}$, Zelin Cui ${ }^{1}$, Rong Tang ${ }^{1}$, Chuanling Zhang ${ }^{2}$ and Qingzhong Liu ${ }^{1 *}$

\begin{abstract}
Background: The data on the prevalence of resistance to mupirocin (MUP), fusidic acid (FA) and retapamulin (RET) in methicillin-resistant Staphylococcus aureus (MRSA) from China are still limited. This study aimed to examine these three antibiotics resistance in 1206 MRSA clinical isolates from Eastern China. Phenotypic MUP, FA and RET resistance was determined by minimum inhibitory concentrations (MICS), and genotypic by PCR and DNA sequencing of the mupA/B, fusB-D, cfr, vgaA/Av/ALC/B/C/E, IsaA-C/E and salA and mutations in ileS, fusA/E, rp/C, and 23S RNA $V$ domain. The genetic characteristics of resistance isolates were conducted by pulsed field gel electrophoresis (PFGE) and multilocus sequence typing (MLST).

Results: Overall MRSA MUP, FA and RET resistance was low (5.1, 1.0 and 0.3\%, respectively). MupA was the mechanism of high-level MUP resistance. All low-level MUP resistance isolates possessed an equivocal mutation N213D in lleS; of these, 2 reported an additional V588F mutation with an impact on the Rossman fold. FusA mutations, such as L461K, H457Q, $\mathrm{H} 457 \mathrm{Y}$ and V90I were the primary FA mechanisms among high-level resistance isolates, most of which also contained fusC; however, all low-level resistance strains carried fusB. Except IsaE gene detected in one isolate, no other resistance mechanisms tested were found among RET-resistant isolates. Additionally, sixteen PFGE types (A-P) were observed, among which type B was the most common (49/76, 64.5\%), followed by types $E$ and $G(4 / 76,5.3 \%$ each) and types $C$ and M (3/76, 3.9\% each). All resistant strains were divided into 15 ST types by MLST. ST764 (24/76, 31.6\%), ST630 (11/76, 14.5\%), ST239 (9/76, 11.8\%) and ST5 (7/76, 9.2\%) were the major types. PFGE type B isolates with the aforementioned STs were mainly found in mupirocin resistant isolates.
\end{abstract}

(Continued on next page)

\footnotetext{
* Correspondence: jiaodamedicine@foxmail.com

tWenjing Chen and Chunyan He contributed equally to this work.

'Department of Clinical Laboratory, Shanghai General Hospital, Shanghai Jiaotong University School of Medicine, 100 Haining Rd, Shanghai 200080, People's Republic of China
}

Full list of author information is available at the end of the article

(c) The Author(s). 2020 Open Access This article is licensed under a Creative Commons Attribution 4.0 International License, which permits use, sharing, adaptation, distribution and reproduction in any medium or format, as long as you give appropriate credit to the original author(s) and the source, provide a link to the Creative Commons licence, and indicate if changes were made. The images or other third party material in this article are included in the article's Creative Commons licence, unless indicated otherwise in a credit line to the material. If material is not included in the article's Creative Commons licence and your intended use is not permitted by statutory regulation or exceeds the permitted use, you will need to obtain permission directly from the copyright holder. To view a copy of this licence, visit http://creativecommons.org/licenses/by/4.0/ The Creative Commons Public Domain Dedication waiver (http://creativecommons.org/publicdomain/zero/1.0/) applies to the data made available in this article, unless otherwise stated in a credit line to the data. 
(Continued from previous page)

Conclusions: MUP, FA and RET exhibited highly activity against the MRSA isolates. Acquired genes and chromosomeborne genes mutations were responsible for MUP and FA resistance; however, the mechanism for some RET-resistant isolates remains to be further elucidated. Also, the surveillance to MUP in MRSA should be strengthened to prevent elevated resistance due to the expansion of clones.

Keywords: Methicillin-resistant Staphylococcus aureus, Mupirocin, Fusidic acid, Retapamulin, Resistance mechanisms, Genetic characteristics

\section{Background}

Methicillin-resistant Staphylococcus aureus (MRSA) is a major pathogen responsible for various hospitalacquired and community-associated infectious worldwide [1]. Because of strong resistance to antibiotics, treatment of MRSA infections is challenging in clinical anti-infective therapy, leading to high risk of mortality and expensive medication [1].

Skin and soft tissue infections (SSTIs) constitute the common diseases caused by $S$. aureus, including MRSA [2], which can be treated with topical antibiotics mupirocin (MUP) and fusidic acid (FA) [3]. However, unreasonable long-term use of these drugs leads to the emergence of resistance, which is a significant public health concern [3]. Therefore, novel topical antimicrobial agent retapamulin (RET) is developed for the treatment of $S$. aureus SSTIs [3].

MUP resistance in staphylococcus is divided into two phenotypes: high-level $(\mathrm{MuH}$, minimum inhibitory concentration $(\mathrm{MIC}) \geq 512 \mu \mathrm{g} / \mathrm{mL}$ ) and low-level (MuL, $\mathrm{MIC}=8-256 \mu \mathrm{g} / \mathrm{mL})[3,4]$. The $\mathrm{MuH}$ is mediated by gene $m u p A$ or $m u p B$, and the MuL is related to point mutations in the chromosomal isoleucyl-tRNA synthetase gene $(i l e S)$ [3, 5]. Previous studies showed that mutations in fus $A$ or fusE in chromosome confer high-level FA resistance (FAH), and acquired fus $B-D$ genes that mediate low-level resistance (FAL) [3, 6, 7]. RET has been licensed in USA and Europe for the topical treatment of SSTIs caused by methicillin-sensitivity $S$. aureus and Streptococcus pyogenes [8]. The RET resistance in S. aureus is often mediated by the point mutations of ribosomal protein L3 (encoded by $r p l C$ ) or the $23 \mathrm{~S}$ rRNA $\mathrm{V}$ domain, or efflux pumps $\mathrm{VgaA} / \mathrm{Av} / \mathrm{A}_{\mathrm{LC}} / \mathrm{B} / \mathrm{C} / \mathrm{E}, \mathrm{LsaA}-\mathrm{C} / \mathrm{E}$ and $\mathrm{SalA}$, or methylation of the $23 \mathrm{~S}$ rRNA subunit (methylated by methyltransferase encoded by chloramphenicol-florfenicol resistance ( $c f r)$ gene) $[3,9,10]$.

Several previous studies reported the resistance of MUP and FA in Eastern China [11-14]. However, since Eastern China is a region with a vast territory, the antibiotic resistance spectrum and the resistance mechanisms may be diversified in different hospitals. Therefore, the data on the resistance of both drugs in clinical isolates of $S$. aureus are limited. To the best of our knowledge, there is no information on RET resistance in China.
In this study, we determined the prevalence of MUP, FA and RET resistance among MRSA isolates from Shanghai and Zhejiang province in Eastern China, and analyzed the underlying resistance mechanisms. Furthermore, PFGE and MLST analysis were also carried out for the genetic characterization of resistant isolates.

\section{Results}

Prevalence of MUP, FA and RET resistance

A total of 1206 MRSA isolates were screened using broth microdilution assay, and $49 \mathrm{MuH}, 12 \mathrm{MuL}, 6$ FAH, 6 FAL, 2 RET-resistant and 1 MuH-RET-resistant isolates were identified. The detailed MIC data of the MUP, FA and RET resistance isolates were listed in Fig. 1. Although $75.1 \%$ isolates in this collection were obtained from respiratory samples, the resistance rates of these strains to MUP, FA and RET did not differ significantly as compared to those obtained from wound secretion, the second most common specimen type (Table 1).

\section{Mechanisms of MUP resistance}

A total of $49 \mathrm{MuH}$ and $1 \mathrm{MuH}-\mathrm{RET}$ resistant isolates contained the mupA gene, and no isolates were mupB positive (Table 2). The sequences of Smr, Mrm and $\mathrm{Lmr}$ DNA fragments were compared to the known ileS gene of S. aureus (Gene bank accession no. X74219). The findings showed that the $12 \mathrm{MuL}$ isolates possessed N213D mutation in the Smr fragment, and $2 \mathrm{MuL}$ isolates had V588F mutation in the Mrm fragment. No mutations were identified in the Lmr fragment. The mutations were accompanied by different MICs: N213D/ V588F, 8 and $32 \mu \mathrm{g} / \mathrm{mL}$ (1 isolate each); N213D, 8 (1 isolate), 32 (2 isolates), 64 ( 1 isolate) and $128 \mu \mathrm{g} / \mathrm{mL}$ (6 isolates) (Fig. 1 and Table 2). There was not significant difference in the distribution of MIC values between isolates with the single mutation $\mathrm{N} 213 \mathrm{D}$ and the double mutation N213D/V588F $(P=0.077)$. In addition, no mupA or mupB gene was detected in MuL isolates.

\section{Mechanisms of FA resistance}

To uncover the mechanisms of FA resistance among 6 FAH isolates, the full-length of fus $A$ and fus $E$ genes were sequenced and compared to those of $S$. aureus ATCC 


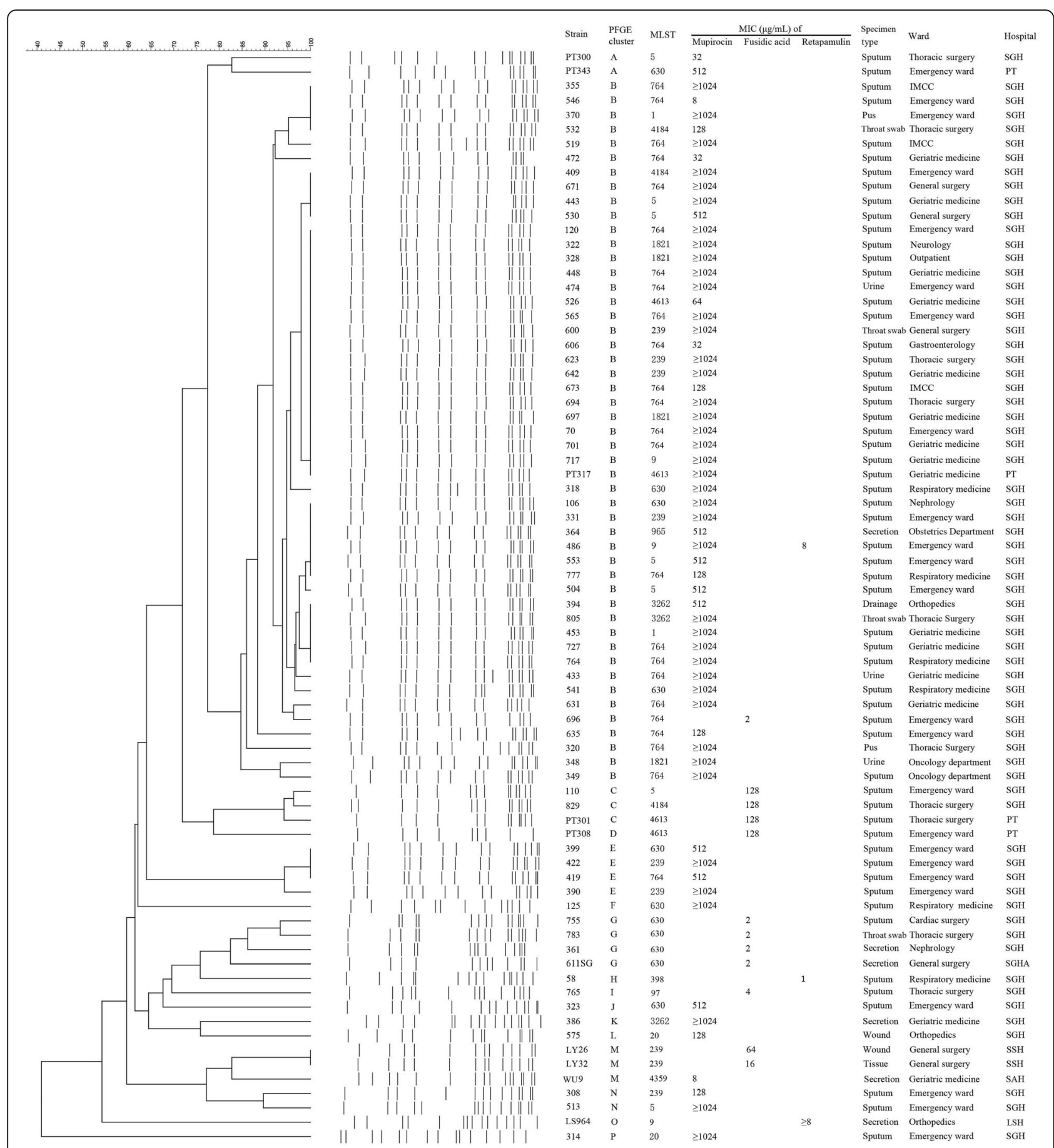

Fig. 1 Molecular characteristics, antibiotic MICs and sources of 76 clinical MRSA isolates with MUP, FA and/or RET resistance. The right panel shown the strain number, PFGE types (isolates with $>80 \%$ similarity were classified into the same type), MLST results, antibiotics MICS (MUP, FA and RET) and strain sources (including sample type, ward and hospital)

25923. Herein, we identified 2 isolates that contained H457 missense mutation (H457Q and H457Y, accompanied by MICs of 128 and $16 \mu \mathrm{g} / \mathrm{mL}$, respectively), while 3 harbored the $\mathrm{L} 461 \mathrm{~K}$ mutation $(\mathrm{MIC}=128 \mu \mathrm{g}$ / $\mathrm{mL})$, and $1(\mathrm{MIC}=64 \mu \mathrm{g} / \mathrm{mL})$ simultaneously possessed L461K, E8K and V90I mutations in FusA (Fig. 1 and
Table 2). All the FA resistance isolates were evaluated for $f u s B, f u s C$ and fusD. Among the $6 \mathrm{FAH}$ isolates, 4 carried the fus $C$ gene. Furthermore, all the $6 \mathrm{FAL}$ isolates were fus $B$-positive, and only one carried fus $C$ gene (Table 2). No mutations were found in fusE, and no isolates were fusD positive. 
Table 1 Resistance of MRSA isolates from respiratory samples and wound secretion to mupirocin, fusidic acid and retapamulin

\begin{tabular}{lllll}
\hline Resistance & No. $(\%)$ of resistant isolates & Wound secretion $(n=200)$ & $X^{2}$ & \\
\cline { 2 - 5 } & Respiratory samples $(n=906)$ & $7(3.5 \%)$ & 1.495 & 0.292 \\
\hline Mupirocin & $51(5.6 \%)$ & $4(2.0 \%)$ & 2.170 & $0.246^{\text {a }}$ \\
Fusidic Acid & $8(0.9 \%)$ & $1(0.5 \%)$ & 0.540 & $0.451^{\text {a }}$ \\
Retapamulin & $2(0.2 \%)$ &
\end{tabular}

a, Fisher's exact test

\section{Mechanism of RET resistance}

$1 / 3$ isolates with resistance to RET harbored the lsaE gene, and the remaining two displayed negative findings for all the resistance mechanisms tested (Fig. 1 and Table 2).

\section{PFGE}

The 76 isolates with MUP, FA and/or RET resistance were divided into 16 patterns: type A-P (Fig. 1). Among $49 \mathrm{MuH}$ alone strains, 40 belonged to type B, 4 were type $\mathrm{E}$, and each of the remaining 5 belonged to types $\mathrm{A}$, $\mathrm{F}, \mathrm{J}, \mathrm{K}$ and $\mathrm{N}$, respectively. One $\mathrm{MuH}-\mathrm{RET}$ resistant isolate was also type $\mathrm{B}$. Among $12 \mathrm{MuL}$ isolates, 9 were type $\mathrm{B}$, and 3 belonged to type $\mathrm{A}, \mathrm{M}$ and $\mathrm{N}$, respectively. Type $\mathrm{C}$ was the most frequent type in FAH strains (3/6; $50 \%)$. Type $\mathrm{G}$ was the most common pattern in FAL strains $(4 / 6 ; 66.7 \%)$. Two MRSA resistant to RET alone belonged to types $\mathrm{O}$ and $\mathrm{H}$, respectively.

\section{MLST}

Fifteen STs were identified among the 76 isolates studied (Fig. 1). ST764 (24/76, 31.6\%) was the most frequent pattern, followed by ST630 (11/76, 14.5\%), ST239 (9/76, $11.8 \%)$ and ST5 (7/76, 9.2\%) and 11 additional STs, namely ST4631 and ST1821 (4/76 each, 5.3\%), ST9, ST3262 and ST4184 (3/76 each, 3.9\%), ST1 and ST20 (2/76 each, 2.6\%), ST965, ST398, ST4359 and ST97 (1/ 76 each, $1.3 \%)$.

\section{Discussion}

MUP is effective for the prevention and treatment of MRSA SSTIs. However, the resistance (including MuL) is beneficial for MRSA treatment and eradication failure $[15,16]$. The prevalence rate of MUP resistance in MRSA clinical isolates varies from $0.5-10.1 \%$ for $\mathrm{MuH}$ and $2.4-8.6 \%$ for MuL in USA, 0-75\% for $\mathrm{MuH}$ and 0$46.7 \%$ for MuL in Asia, and from $0.8-98 \%$ for $\mathrm{MuH}$ and 0-31.2\% for MuL in Europe [15]. In the present study, the isolation rates of $\mathrm{MuH}$ and $\mathrm{MuL}$ were low: $4.1 \%$ (50/ $1206)$ and $1.0 \%(12 / 1206)$, respectively. Recent studies displayed that the prevalence of $\mathrm{MuH}$ is mediated by plasmid-borne mupA gene [15], which is the same as our results. Although $m u p B$, also a plasmid-borne gene, is correlated with $\mathrm{MuH}$ [5], the mechanism is rarely examined in staphylococci, including the isolates investigated in this study. The point mutations in the ileS gene, resulting in amino changes in MUP-binding site (located in amino acids 450-650, also named Rossman fold), are the main mechanisms determining MuL $[15,17]$. V588F and V631F are well identified frequent mutations in IleS responsible for MuL [15]. In this study, only two MuL isolates (PT300 and wu9) contained the V588F mutation, and no MuL isolates harbored the V631F mutation. Notably, all MuL isolates harbored the $\mathrm{N} 213 \mathrm{D}$ mutation that was located in a hotspot amino acid sequence between 200 and 350, as described by Lee et al. [17]. The N213D mutation has been previously reported and are considered to have no impact on the sensitivity of MUP [18]. Although the mupA gene located on the chromosome is also associated with $\mathrm{MuL}$ [3], we did not detect the gene in our MuL isolates. Also, no other mutations in IleS were found. Lee et al. [17] reported that a mutation of S634F that confers phenotype of susceptibility or MuL in diverse isolates. In view of the above reasons, the contribution of N213D mutation to MuL should be evaluated further.

FA is a steroidal antimicrobial agent that suppresses the production of bacterial proteins by stopping the dissociation of elongation factor $\mathrm{G}$ (EF-G) from ribosome $[6,19]$. Clinically, the main application of topical FA is for the treatment of SSTIs and decolonization of S. aureus, including MRSA; this method is similar to that of MUP [3]. The prevalence of FA resistance reported by recent large studies varies in MRSA isolates from USA (0-0.3\%), Australian (4.1-5.1\%), Denmark (17.8\%), Greece (57.0\%) and other European countries (9.9\%) [3]. In China, the resistance levels in MRSA are also different in different areas, for example, 3.0-5.3\% in MRSA from Beijing, Shanghai, Shenyang and Shenzhen cities [12, 20], and $27.1 \%$ in MRSA from Wenzhou city [11]. Compared to the aforementioned data from China, our results showed a very low resistance rate $(12 / 1206,1.0 \%)$.

In $S$. aureus, the mutations in fusA (encoding EF-G) or fusE (coding for ribosome protein L6, RplF) lead to a decreased affinity of FA for the EF-G ribosome complex [3, 21]. About $>30$ point mutations in FusA sequence were described; however, only a few were experimentally verified to play a role in FA resistance $[3,22,23]$. The V90I mutation in domain I (amino acids 1-280) and H457Y, H457Q and L461K 


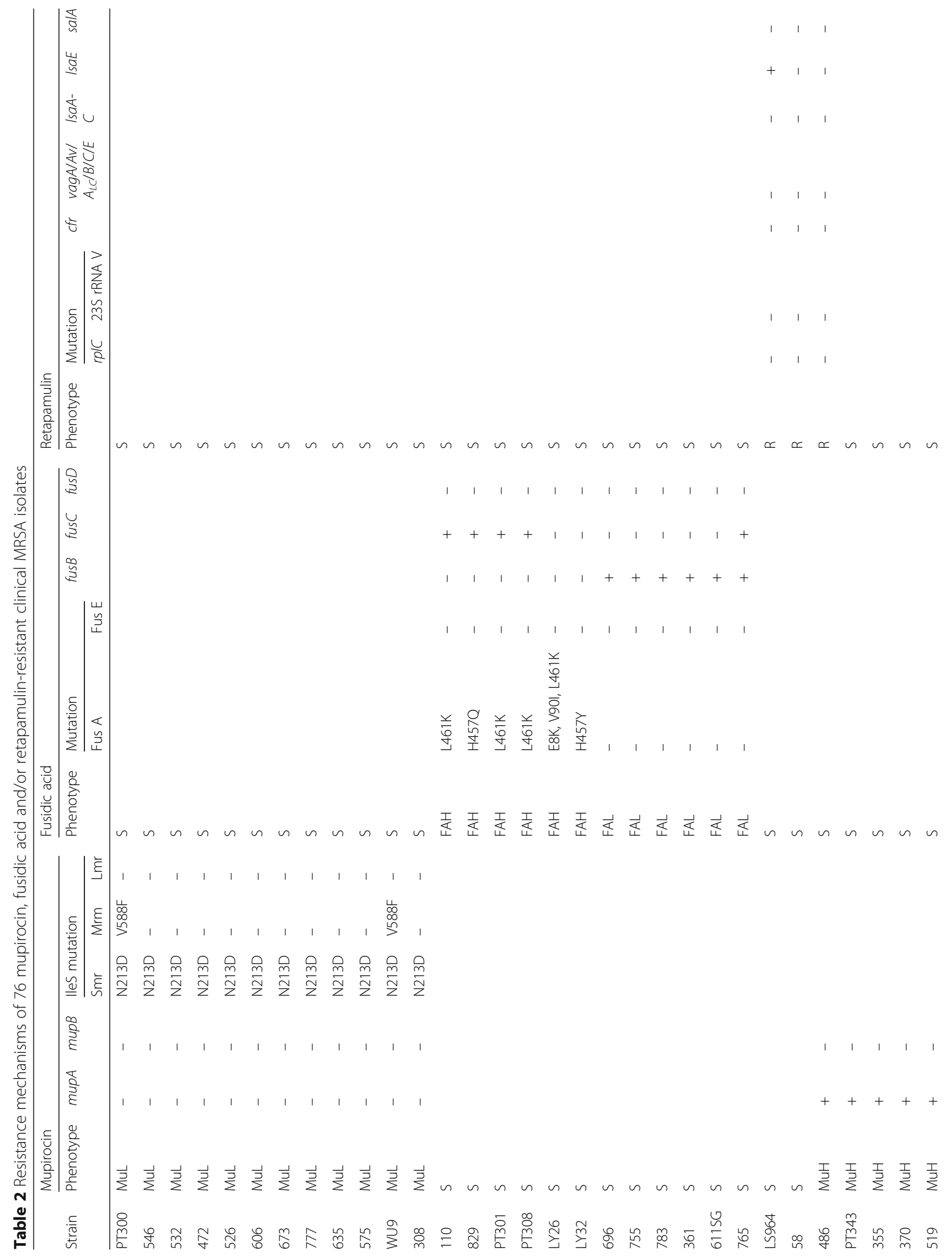




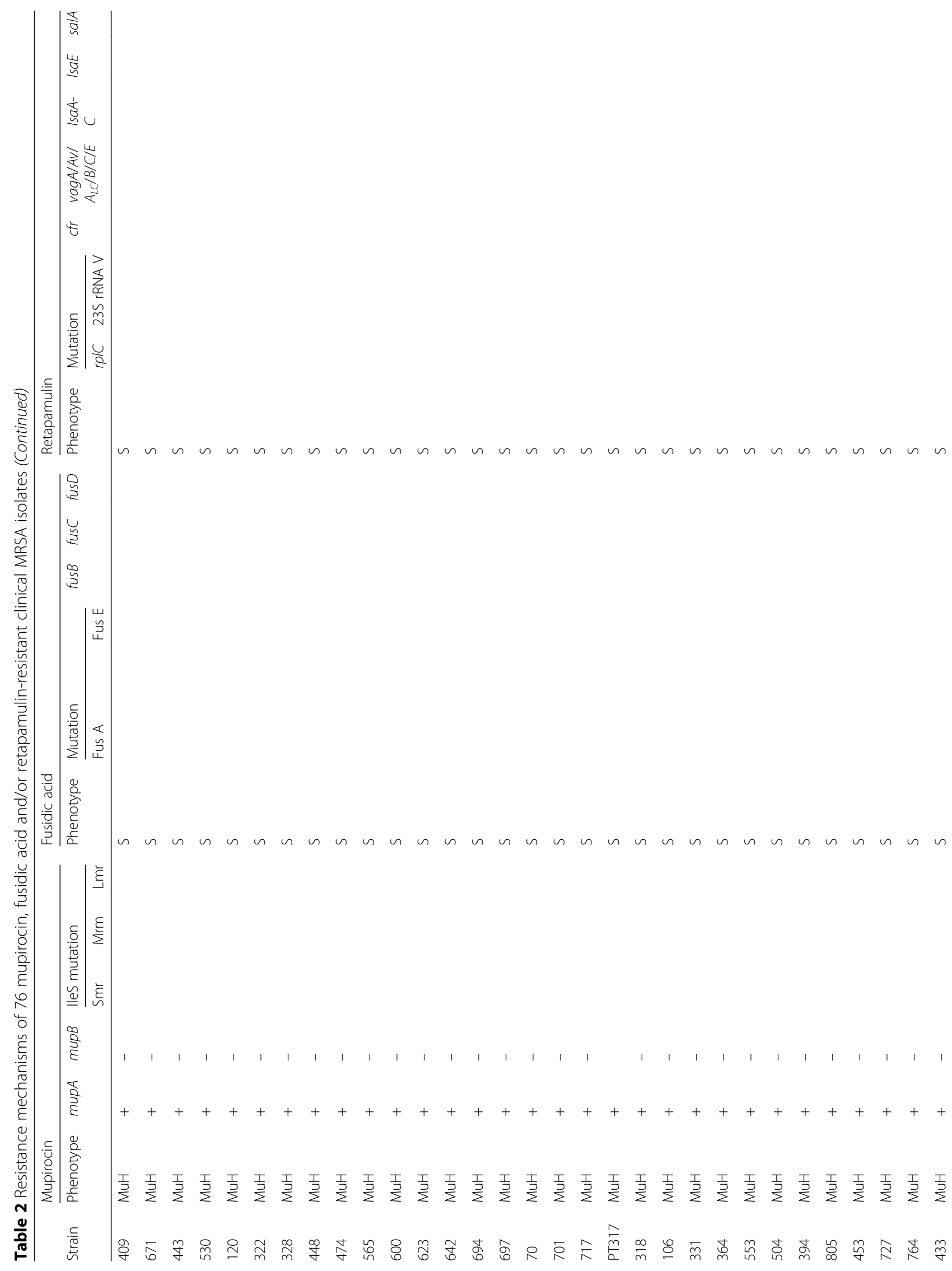




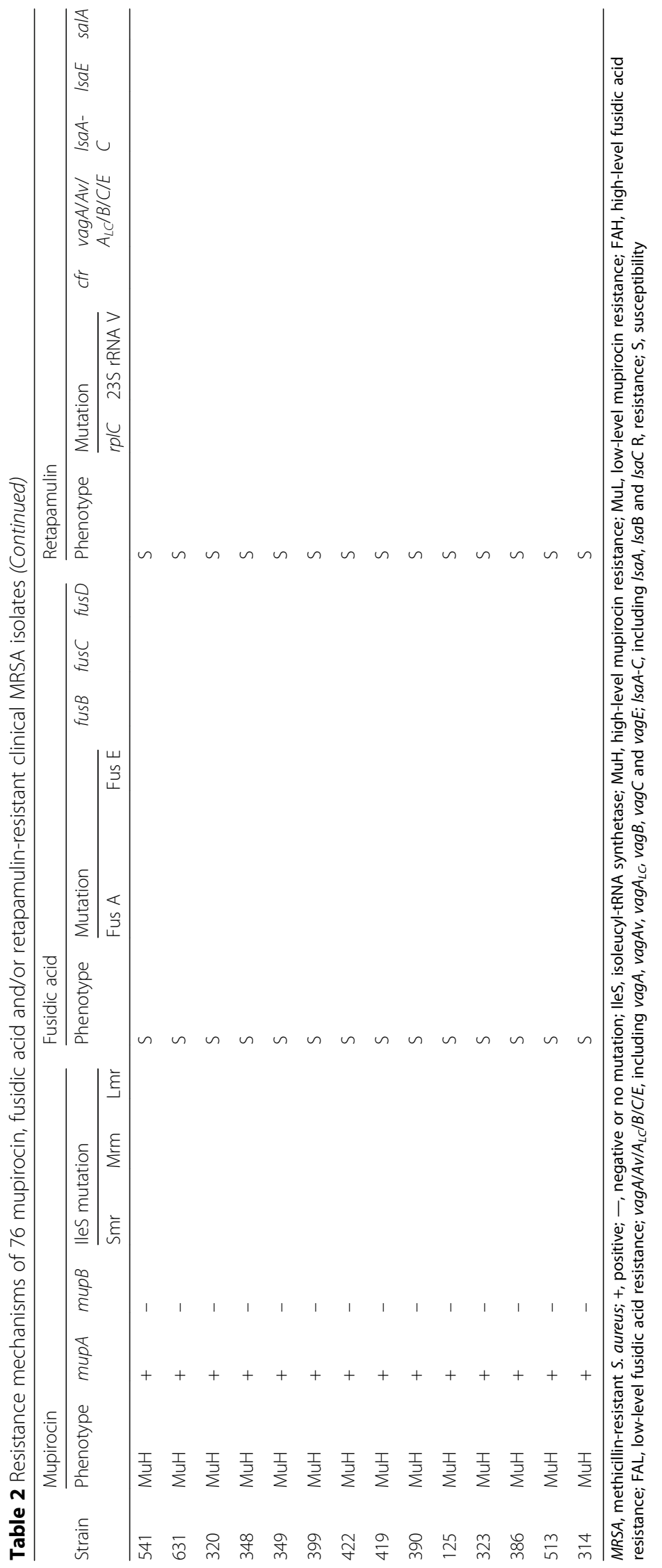


mutations in domain III (amino acids 404-480) were observed in this study, and have been previously identified causing FAH in $S$. aureus [21, 22, 24]; also, L461K is the most prevalent mechanism among clinical FAH S. aureus strains [3]. L461K also existed in the majority $(4 / 6,80 \%) \mathrm{FAH}$ isolates, leading to FA MICs $\geq 64 \mu \mathrm{g} / \mathrm{mL}$ in the current study. One novel substitution with E8K in domain I was identified in addition to V90I and L461K mutations in the FAH isolate. However, whether the novel mutation is associated with FA resistance is not yet clarified.

Protection of EF-G by FusB family molecules is another mechanism conferring the resistance (low-level) of FA [3]. FusB family proteins (including FusB, FusC, and FusD) can restore the translation of protein by binding to EF-G in the presence of FA [3]. Previous studies showed that $f u s B$ was the most prevalent in Netherlands and mainland China [11, 12, 25], and fusC primarily existed in isolates from Taiwan, Australia, USA and European [26-28]. In our isolates with FA resistance, fusB existed in all FAL isolates (6/6), and fusC was most prevalent in FAH isolates $(4 / 6,66.7 \%)$. The fusD gene was identified in Staphylococcus saprophyticus. The gene is related to the "intrinsic resistance of FA" among this species [19]. Hitherto, this determinant is rarely detected in S. aureus strains.

RET is a semisynthetic pleuromutilin drug that represses the synthesis of bacterial proteins by interacting with domain $\mathrm{V}$ of $50 \mathrm{~S}$ ribosomal subunit [3]. This drug has a potency to act as an alternative to MUP to eradicate the $S$. aureus colonization, except when used for the treatment of $S$. aureus-induced SSTIs [8, 29]. Currently, limited data are available among clinical $S$. aureus strains worldwide. The resistance rates of 664 UK $S$. aureus (74\% are MRSA), 155 USA MRSA, 403 USA MRSA, and 400 USA $S$. aureus from several studies were $0.15,2.6$, 0.25 , and $9.5 \%$, respectively $[8,29-31]$. In this study, the prevalence of RET resistance was very low (3/1206, $0.24 \%)$. In the UK and USA, the RET resistance among $S$. aureus or MRSA with MUP resistance was $<1-2.6 \%$ $[8,31]$. In the present study, only one MRSA isolate was observed to have resistance to both RET and MUP. For RET resistance in staphylococci, except mutation and/or methylation of ribosomal protein and rRNA, the ABC-F proteins (efflux pumps) encoded by three types of genes (vga, lsa and sal) were alsocritical mechanisms [32]. However, in our 3 RET-resistant isolates, only one was confirmed to contain the salE gene. The salE gene confers the resistance to pleuromutilins, lincosamides and streptogramin A [32]. Fortunately, the gene was localized on the chromosome or non-conjugative plasmid (pV7073) [32], which limits its transmission among staphylococci. For two strains we could not clarify phenotypic resistance to the detected resistance determinants, implying that there possibly exists other gene or gene variant leading to RET resistance.

ST239 and ST5 are two predominant sequence types in China. However, the strains identified in this study mainly belonged to ST764 (31.6\%), which was more than the total percentage of ST239 and ST5 (16/76, 21.1\%). Early investigation showed that most of FA-resistant MRSA belong to ST239 and ST5 [7, 27]. However, our findings showed no FA-resistant strains were these two types. ST764 MRSA, first reported in Japan, is a single-locus of ST5 nosocomial MRSA clone with or without the arginine catabolic mobile element (ACME, a feature of CA-MRSA) $[33,34]$. In recent years, several studies reported the $S$. aureus clone with ST764 in China [13, 35]. In this study, ST630 (14.5\%) was the second most common type, which also became a prevalent $S$. aureus clone causing SSTIs in Wenzhou region, Zhejiang province, China [36]. Figure 1 shows that $66.7 \%$ (4/6) FAL isolates belonged to ST630 (PFGE G-ST630), which was similar to the previous report [9]. Notably, 50\% (6/12) MUL isolates and 39.0\% (16/41) MUH isolates with MIC $\geq 1024 \mu \mathrm{g} / \mathrm{mL}$ were PFGE BST764, and were identified from the same hospital (Shanghai General Hospital) (Fig. 1), indicating clone transmission. In addition, the spread of other MRSA clones with different genetic patterns, such as PFGE BST1821, PFGE B-ST239 (4 isolates each), PFGE B-ST5 and PFGE B-ST630 (3 isolates each) were also responsible for the increased MUH in this hospital. It should be noted that some resistant isolates had the same PFGE pattern (such as PFGE B, C, E, G or M), although the ST types were diverse from each other (Fig. 1). These finding hint that these isolates might be clonally related.

\section{Conclusions}

In this study, the MRSA isolates exhibited a low prevalence of resistance to MUP, FA and RET, especially to the latter two, and cross-resistance to the three antibiotics was rare. The mupA gene mechanism mediated $\mathrm{MuH}$. The contribution of the N213D mutation in IleS found in our MuL isolates that decreased the resistance of MUP is yet unclear. FusA mutations, FusB and FusC were the frequent genetic mechanisms that mediate FA resistance. Phylogenetic detection showed the transmission of multiple clones, especially PFGE B-ST764 clone that made a major contribution to the increased MUP resistance in this collection of isolates. Owing to the concern of resistance development and clonal dissemination in healthcare, continuous surveillance for the resistance of these topic antibiotics in S. aureus is essential in China.

\section{Methods}

\section{Bacterial isolates}

A total of 1206 non-duplicate MRSA isolates from various clinical specimens were collected from 8 hospitals in 
Table 3 Primers and PCR conditions for detecting mupirocin, fusidic acid and retapamulin resistance genes in this study

\begin{tabular}{ccc}
\hline Gene & Primer name & Primer sequence $\left(5^{\prime}-3^{\prime}\right)$ \\
\hline Molecular mechanisms related to mupirocin resistance \\
mupA & mupA-F & TATATTATGCGATGGAAGGTTGG \\
& mupA-R & AATAAAATCAGCTGGAAAGTGTTG \\
mupB & mupB-F & CTAGAAGTCGATTTGGAGTAG \\
& mupB-R & AGTGTCTAAAATGATAAGACGATC
\end{tabular}
PCR amplification program Size Reference

iles (including the following 3 fragments)

$\begin{array}{lll}S m r & S m r-\mathrm{F} & \text { ATAAAGGTAAAAAGCCAGTTTATTGGT } \\ & S m r-\mathrm{R} & \text { TAATCGCAACATTTGATGGAATTGTC } \\ \mathrm{Mrm} & \mathrm{Mrm}-\mathrm{F} & \text { TCCCAGCAGATATGTATTAGAAGGT } \\ & \mathrm{Mrm}-\mathrm{R} & \text { AACCACTTGGTCAGGTACAATCACA } \\ \mathrm{Lmr} & L m r-\mathrm{F} & \text { GTAAATCTTAGGTAATGTGATTGTAC } \\ & L m r-\mathrm{R} & \text { TCTTCTTAACATGTGGTGTATGAGA }\end{array}$

Initial pre-denaturation at $94^{\circ} \mathrm{C}$ for $5 \mathrm{~min} ; 30$ cycles of denaturation ( $30 \mathrm{~s}$ at $\left.94^{\circ} \mathrm{C}\right)$, annealing $(30 \mathrm{~s}$; at $53^{\circ} \mathrm{C}$ for mupA, and $55^{\circ} \mathrm{C}$ for mupB, Smr. Mrm and $L m r)$, and extension $\left(50 \mathrm{~s}\right.$ at $\left.72^{\circ} \mathrm{C}\right)$, followed by a final extension step of $7 \mathrm{~min}$ at $72^{\circ} \mathrm{C}$

$457 \mathrm{bp}$ 5

$674 \mathrm{bp}$

$200 \mathrm{bp}$ 38

$450 \mathrm{bp}$

$690 \mathrm{bp}$

Molecular mechanisms associated with fusidic acid resistance

$\begin{array}{lll}\text { fusA } & \text { fusA-F } & \text { TTACCCTGAGTGTGTTCT } \\ & \text { fusA-R } & \text { TACATTAAGCTCACCTTGT } \\ \text { fusB } & \text { fusB-F } & \text { TCATATAGATGACGATATTG } \\ & \text { fusB-R } & \text { ACAATGAATGCTATCTCGAC } \\ \text { fusC } & \text { fusC-F } & \text { GATATTGATATCTCGGACTT } \\ & \text { fusC-R } & \text { AGTTGACTTGATGAAGGTAT } \\ \text { fusD } & \text { fusD-F } & \text { TGCTTATAATTCGGTCAACG } \\ & \text { fusD-R } & \text { TGGTTACATAATGTGCTATC } \\ \text { fusE } & \text { fusE-F } & \text { CCTAGTGACGTAACAGTAAC } \\ & \text { fusE-R } & \text { CGGCGWACRTATTCACCTTG }\end{array}$

Molecular mechanisms associated with retapamulin resistance

\begin{tabular}{|c|c|c|}
\hline \multirow[t]{2}{*}{ rp/C } & $r p / C-F$ & AACCTGATTTAGTTCCGTCTA \\
\hline & $r p / C-R$ & GTTGACGCTITAATGGGCTTA \\
\hline \multirow[t]{2}{*}{$c f r$} & $c f r-F$ & GAGATAACAGATCAAGTTITA \\
\hline & $c f r-R$ & CGAGTATATTCATTACCTCAT \\
\hline \multirow[t]{2}{*}{ vgaA } & vgaA-F & TCACATGATCGCGCTIITITAGAT \\
\hline & $v g a A-R$ & TCGCTCTCCACCACTTAAGACACT \\
\hline \multirow[t]{2}{*}{$v g a A v$} & $v g a A v-F$ & CTCTITGTACGAGTATATGG \\
\hline & $v g a A v-R$ & GTTTCTTAGTAGCTCGTTGAGC \\
\hline \multirow[t]{2}{*}{$v g a A_{L C}$} & $\operatorname{vgaA}_{L C}-\mathrm{F}$ & CATTATCGCCATCTGTCA \\
\hline & $\operatorname{vgaA}_{L C}-\mathrm{R}$ & AATTCTTCCGAAGGTTCA \\
\hline \multirow[t]{2}{*}{$\operatorname{vgaB}$} & $v g a B-F$ & TGACAATATGAGTGGTGGTG \\
\hline & $v g a B-R$ & GCGACCATGAAATTGCTCTC \\
\hline \multirow[t]{2}{*}{ vgaC } & vgaC-F & CGTATGCCCAGAGTGAG \\
\hline & vgaC-R & GAGTGCTTCCGTATCCA \\
\hline \multirow[t]{2}{*}{ vgaE } & $v g a E-F$ & GAAATATGGGAAATAGAAGATGG \\
\hline & vgaE-R & TGATTCTCTAACCACTCTTC \\
\hline \multirow[t]{2}{*}{ IsaA } & IsaA-F & ACCGTGAAGGTGATAAGT \\
\hline & IsaA-R & TTGGGTGTAATCTAACTGAT \\
\hline \multirow[t]{2}{*}{$I s a B$} & IsaB-F & TCCACTGCCGTTCTITCC \\
\hline & IsaB-R & AGCCATGTACCGTCCTIT \\
\hline
\end{tabular}

Initial pre-denaturation at $94^{\circ} \mathrm{C}$ for $5 \mathrm{~min} ; 30$ cycles of denaturation $\left(30 \mathrm{~s}\right.$ at $\left.94^{\circ} \mathrm{C}\right)$, annealing $(30 \mathrm{~s}$; at $50^{\circ} \mathrm{C}$ for vgaA and $v g a A_{L C}, 52^{\circ} \mathrm{C}$ for vgaB, vagC, vgaE, IsaA-C and $I s a E$, and $55^{\circ} \mathrm{C}$ for $r p / C$, cfr, vgaAv, salA and $23 S$ RNA V), and extension ( 2 min, at $72^{\circ} \mathrm{C}$ for vgaC, vgaE and salA; $50 \mathrm{~s}$, at $72^{\circ} \mathrm{C}$ for other genes), followed by a final extension step of 7 min at $72^{\circ} \mathrm{C}$

Initial pre-denaturation at $94^{\circ} \mathrm{C}$ for $5 \mathrm{~min} ; 30$ cycles of denaturation ( $30 \mathrm{~s}$ at $94^{\circ} \mathrm{C}$ ), annealing ( $30 \mathrm{~s}$; at $53^{\circ} \mathrm{C}$ for fusB-D and fusE, and $40^{\circ} \mathrm{C}$ for fusA), and extension $\left(2 \mathrm{~min}\right.$, at $72^{\circ} \mathrm{C}$ for fus $\mathrm{A} ; 50 \mathrm{~s}$, at $72^{\circ} \mathrm{C}$ for other genes), followed by a final extension step of 7 min at $72{ }^{\circ} \mathrm{C}$

$2250 \mathrm{bp}$ 7

496 bp $\quad 26$

$128 \mathrm{bp}$

$525 \mathrm{bp}$

$505 \mathrm{bp}$

822 bp 8

$1050 \mathrm{bp}$ 39

$770 \mathrm{bp}$

29

$770 \mathrm{bp}$

40

$541 \mathrm{bp}$

9

$577 \mathrm{bp}$

$1073 \mathrm{bp}$

995 bp

500 bp

715 bp 
Table 3 Primers and PCR conditions for detecting mupirocin, fusidic acid and retapamulin resistance genes in this study (Continued)

\begin{tabular}{|c|c|c|c|c|c|}
\hline Gene & Primer name & Primer sequence $\left(5^{\prime}-3^{\prime}\right)$ & PCR amplification program & Size & Reference \\
\hline \multirow[t]{2}{*}{ IsaC } & IsaC-F & GGCTATGTAAAACCTGTATTTG & & $429 \mathrm{bp}$ & \\
\hline & Isac-R & ACTGACAATTITCTTCCGT & & & \\
\hline \multirow[t]{2}{*}{ IsaE } & IsaE-F & TTGTACGGAATGTATGG & & $675 \mathrm{bp}$ & \\
\hline & IsaE-R & TTCGCTTCTATTAAGCACTCTT & & & \\
\hline \multirow[t]{2}{*}{ salA } & salA-F & CGATGAACCAACAAACCACA & & $981 \mathrm{bp}$ & 10 \\
\hline & salA-R & AGGACCGAACCTTGAAATGA & & & \\
\hline \multirow[t]{2}{*}{ 23S RNA V } & 23S RNA-F & TGGGCACTGTCTCAACGA & & $634 \mathrm{bp}$ & 41 \\
\hline & 23S RNA-R & GGATAGGGACCGAACTGTCTC & & & \\
\hline MLST typing & & & & & 43 \\
\hline \multirow[t]{2}{*}{$\operatorname{arcc}$} & $\operatorname{arcC}-\mathrm{F}$ & TTGATTCACCAGCGCGTATTGTC & \multirow{14}{*}{$\begin{array}{l}\text { Initial pre-denaturation at } 94^{\circ} \mathrm{C} \text { for } 5 \text { min; } 30 \text { cycles } \\
\text { of denaturation }\left(30 \mathrm{~s} \text { at } 94^{\circ} \mathrm{C}\right) \text {, annealing }(30 \mathrm{~s} \text {; at } \\
55^{\circ} \mathrm{C} \text { for } 7 \text { house-keeping genes), and extension } \\
\left(50 \mathrm{~s} \text {, at } 72^{\circ} \mathrm{C} \text { for other genes), followed by a final }\right. \\
\text { extension step of } 7 \text { min at } 72^{\circ} \mathrm{C}\end{array}$} & $456 \mathrm{bp}$ & \\
\hline & $\operatorname{arcC}-\mathrm{R}$ & AGGTATCTGCTTCAATCAGCG & & & \\
\hline \multirow[t]{2}{*}{$\operatorname{aroE}$} & $\operatorname{aroE}-\mathrm{F}$ & ATCGGAAATCCTATTTCACATTC & & $456 \mathrm{bp}$ & \\
\hline & $\operatorname{aroE}-\mathrm{R}$ & GGTGTTGTATTAATAACGATATC & & & \\
\hline \multirow[t]{2}{*}{ glpF } & $g l p F-F$ & CTAGGAACTGCAATCTTAATCC & & $465 \mathrm{bp}$ & \\
\hline & glpF-R & TGGTAAAATCGCATGTCCAATTC & & & \\
\hline \multirow[t]{2}{*}{ gmk } & $g m k-F$ & ATCGTITTATCGGGACCATC & & $429 \mathrm{bp}$ & \\
\hline & $g m k-R$ & TCATTAACTACAACGTAATCGTA & & & \\
\hline \multirow[t]{2}{*}{ pta } & pta-F & GTTAAAATCGTATTACCTGAAGG & & $474 \mathrm{bp}$ & \\
\hline & pta-R & GACCCTITTGTTGAAAAGCTTAA & & & \\
\hline \multirow[t]{2}{*}{ tpi } & tpi-F & TCGTTCATTCTGAACGTCGTGAA & & 402 bp & \\
\hline & tpi-R & TTTGCACCTTCTAACAATTGTAC & & & \\
\hline \multirow[t]{2}{*}{ yqil } & yqil-F & CAGCATACAGGACACCTATTGGC & & 516 bp & \\
\hline & yqiL-R & CGTTGAGGAATCGATACTGGAAC & & & \\
\hline
\end{tabular}

Shanghai and Zhejiang province, Eastern China (Shanghai General Hospital (1037 isolates from July 2010 to June 2015), Ruijin Hospital (22 isolates during January 2011 to December 2011), Shanghai Sixth People's Hospital (36 isolates between Decermber 2010 and Decermber 2012), Shanghai People's Hospital of Putuo District (45 isolates from January 2013 to May 2014), Shuguang Hospital Affiliated to Shanghai University of Traditional Chinese Medicine (21 isolates from February 2014 to September 2014), Shanghai Armed Police Corps Hospital (9 isolates from January 2014 to June 2014), Zhejiang Xiaoshan Hospital (8 isolates from March 2012 to October 2012) and The Central Hospital of Lishui City, Zhejiang Province (28 isolates, July 2013 to September 2014)). The majority of these isolates were obtained from respiratory samples $(906 / 1206,75.1 \%)$ and wound secretion (200/1206, 16.6\%). The MRSA isolates were frequently detected in intensive care units, respiratory medicine, geriatric medicine, thoracic surgery and nephrology wards causing maximal impact. All isolates were identified using VITEK microbial identification system (bioMérieux, Marcy l' Etoile, France). The resistance to methicillin was detected with a $30 \mu \mathrm{g}$ cefoxitin disk (Oxoid, Basingstoke, UK) [4]. MuH (MIC $\geq 512 \mu \mathrm{g} / \mathrm{mL}$ ),
MuL $($ MIC $=8-256 \mu \mathrm{g} / \mathrm{mL})$, FAH $($ MIC $\geq 8 \mu \mathrm{g} / \mathrm{mL})$, FAL $(\mathrm{MIC}=2-4 \mu \mathrm{g} / \mathrm{mL})$ and RET resistance $(\mathrm{MIC} \geq 1 \mu \mathrm{g} / \mathrm{mL}$ was defined as resistance in this study) were screened from all the isolates collected by broth microdilution method $[4,6,14,37]$. For three-antibiotic (MUP, FA and RET) resistant isolates, the methicillin resistance was further confirmed by the amplification of mecA and $m e c C$ genes [35]. MUP, FA and RET were purchased from Shanghai Boyle Chemical Co., Ltd., China. Cationadjusted Mueller-Hinton broth was produced by Shanghai Comagal Microbial Technology Co., Ltd., China. S. aureus ATCC 25923 and ATCC 29213 were used as quality control strains for antibiotic susceptibility testing.

\section{Total DNA extraction}

The cultures of MRSA with MUP, FA and/or RET resistance were incubated with lysostaphin $(1 \mathrm{mg} / \mathrm{mL})$ (Sangon Biotech, Shanghai, China) at $37^{\circ} \mathrm{C}$ for $0.5 \mathrm{~h}$. Then, the DNA was extracted according to the instructions of the bacterial genomic DNA kit (Tiangen Biotech, Beijing, China), and utilized as a template for PCR assays. 
Detection of MUP, FA, and RET resistance genes

PCR was used to detect the factors mediating MUP (mupA, тupB and ileS (amplifying three fragments of $S m r, M r m$ and $L m r$, which might possess the mutations mediating mupirocin resistance)), FA (fus $A$, fus $B$, fus $C$, fusD and fusE) and RET (rplC, cfr, vgaA/Av/A $A_{L C} / B / C / E$, lsaA-C/E, salA and 23S RNA V) resistance [5, 7-10, 26, $29,38-41]$. The primers and programs for the amplification of genes are presented in Table 3. The DNA sequencing of one randomly selected PCR product for mupA, тupB, fusB-D, cfr, vgaA/Av/A $A_{L C} / B / C / E$, lsaA-C/ $E$, and salA was used for the identification of target fragments. All the PCR products for ileS-Smr, ileS-Mrm, ileS-Lmr, fusA, fusE, rplC and 23S RNA V were sequenced to determine the putative mutations.

\section{Pulsed field gel electrophoresis (PFGE)}

PFGE was performed for MUP, FA and/or RET resistant strains, as described previously [42]. BioNumerics software 7.0 was used for the analysis of DNA fingerprint profiles. An $80 \%$ cutoff value was set to assess the similarity.

\section{Multilocus sequence typing (MLST)}

MLST was conducted by sequencing of the internal fragments of the 7 housekeeping genes, $\operatorname{arcC}$, aroE, $g l p F$, gmk, pta, tpi and yqiL on MUP, FA and/or RETresistant MRSA using the primers (Table 3), as described previously [43]. Sequence types (STs) were determined based on the data from the MLST database for S. aureus (http://saureus.mlst.net/).

\section{Statistical analysis}

The difference of MUP, FA and RET-resistant rates of isolates from major samples was analyzed by Pearson's Chi-square test or Fisher's exact test using SAS 8.0 (SAS Institute, Cary, NC, USA). Student's t test was used to determine the difference in the distribution of MIC values between isolates with different mutations. A $P$ value $<0.05$ (two-tailed) indicated significance.

\footnotetext{
Abbreviations

FA: Fusidic acid; FAH: High-level fusidic acid resistance; FAL: Low-level fusidic acid resistance; IleS: Isoleucyl-tRNA synthetase; LSH: The Central Hospital of Lishui City, Zhejiang Province; MIC: Minimum inhibitory concentration; MLST: Multilocus sequence typing; MRSA: Methicillin-resistant Staphylococcus aureus; MuH: High-level mupirocin resistance; MuL: Low-level mupirocin resistance; MUP: Mupirocin; PCR: Polymerase chain reaction; PFGE: Pulsedfield gel electrophoresis; PT: Shanghai People's Hospital of Putuo District; RET: Retapamulin; SAH: Shanghai Armed Police Corps Hospital; SGH: Shanghai General Hospital; SSH: Shanghai Sixth People's Hospital; SGHA: Shuguang Hospital Affiliated to Shanghai University of Traditional Chinese Medicine
}

\section{Acknowledgments}

We would like to thank all of the researchers for their contributions to the present study. We thank Dr. Shu Jin (Shanghai People's Hospital of Putuo District) for providing the methods for the lysis of S. aureus (patent number:
ZL201310124795.2), and Lizhong Han, Jing Tang and Jinwei Huang for kindly supplying the MRSA clinical isolates.

\section{Authors' contributions}

QZL designed and conceived the investigation; WJC and CYH carried out the experiments; WJC, CYH, HY, RT, ZLC, WS and CLZ analyzed the data; WJC and QZL wrote and revised this manuscript. All authors had read and agreed with the final manuscript.

\section{Funding}

This study was supported by grants from the National Natural Science Foundation of China (No.81772247 and No.81371872), Shanghai Jiaotong University Medical-Engineering Cross Research Youth Fund (YG2016QN31), and Medical Science and Technology Plan (Young Talents) of Zhejiang province (2019RC256). The funders had no role in the study design and collection, analysis and interpretation of data and in the preparation of the manuscript.

\section{Availability of data and materials}

All data generated or analyzed during this study are included in this article.

Ethics approval and consent to participate

Not applicable.

\section{Consent for publication}

Not applicable.

\section{Competing interests}

The authors declare that they have no competing interests.

\section{Author details}

'Department of Clinical Laboratory, Shanghai General Hospital, Shanghai Jiaotong University School of Medicine, 100 Haining Rd, Shanghai 200080, People's Republic of China. ${ }^{2}$ Department of Clinical Laboratory, Xiaoshan Hospital, Hangzhou, Zhejiang Province, China.

Received: 20 January 2020 Accepted: 16 June 2020

Published online: 29 June 2020

\section{References}

1. Turner NA, Sharma-Kuinkel BK, Maskarinec SA, Eichenberger EM, Shah PP, Carugati M, MIC Holland TL, Fowler VG Jr. Methicillin-resistant Staphylococcus aureus: an overview of basic and clinical research. Nat Rev Microbiol. 2019;17(4):203-18.

2. Bassetti M, Carnelutti A, Righi E. The role of methicillin-resistant Staphylococcus aureus in skin and soft tissue infections. Curr Opin Infect Dis. 2017;30(2):150-7.

3. Williamson DA, Carter GP, Howden BP. Current and emerging topical antibacterials and antiseptics: agents, action, and resistance patterns. Clin Microbiol Rev. 2017;30(3):827-60.

4. Clinical and Laboratory Standards Institute. Performance standards for antimicrobial susceptibility testing, 29th informational supplement (M100S29). Wayne, PA: Clinical and Laboratory Standards Institute; 2019.

5. Seah C, Alexander DC, Louie L, Simor A, Low DE, Longtin J, Melano RG. MupB, a new high-level mupirocin resistance mechanism in Staphylococcus aureus. Antimicrob Agents Chemother. 2012:56(4):1916-20.

6. The European Committee on Antimicrobial Susceptibility Testing Breakpoint Tables for Interpretation of MICs and Zone Diameters. Available online: http://www.eucast.org/clinical_breakpoints/. Accessed 10 Nov 2016.

7. Chen HJ, Hung WC, Tseng SP, Tsai JC, Hsueh PR, Teng LJ. Fusidic acid resistance determinants in Staphylococcus aureus clinical isolates. Antimicrob Agents Chemother. 2010;54(12):4985-91.

8. Woodford N, Afzal-Shah M, Warner M, Livermore DM. In vitro activity of retapamulin against Staphylococcus aureus isolates resistant to fusidic acid and mupirocin. J Antimicrob Chemother. 2008;62(4):766-8.

9. Li J, Li B, Wendlandt S, Schwarz S, Wang Y, Wu C, Ma Z, Shen J. Identification of a novel $\mathrm{vga}(\mathrm{E})$ gene variant that confers resistance to pleuromutilins, lincosamides and streptogramin a antibiotics in staphylococci of porcine origin. J Antimicrob Chemother. 2014;69(4):919-23.

10. Wendlandt S, Kadlec K, Feßler AT, Schwarz S. Identification of ABC transporter genes conferring combined pleuromutilin-lincosamide- 
streptogramin a resistance in bovine methicillin-resistant Staphylococcus aureus and coagulase-negative staphylococci. Vet Microbiol. 2015;177(3-4): 353-8.

11. Yu F, Liu Y, Lu C, Lv J, Qi X, Ding Y, Li D, Huang X, Hu L, Wang L. Dissemination of fusidic acid resistance among Staphylococcus aureus clinical isolates. BMC Microbiol. 2015;15:210.

12. Huang J, Ye M, Ding H, Guo Q, Ding B, Wang M. Prevalence of fus $B$ in Staphylococcus aureus clinical isolates. J Med Microbiol. 2013;62(Pt 8):1199_ 203.

13. Chen S, Jin Y, Lin C, Hao Z, Duan J, Guo Y, Wang S, Hu L, Wang L, Yu F. Low prevalence of mupirocin resistance among Staphylococcus aureus clinical isolates from a Chinese tertiary hospital. J Med Microbiol. 2019;68(2):201-5.

14. Liu QZ, Wu Q, Zhang YB, Liu MN, Hu FP, Xu XG, Zhu DM, Ni YX. Prevalence of clinical meticillin-resistant Staphylococcus aureus (MRSA) with high-level mupirocin resistance in Shanghai and Wenzhou. China Int J Antimicrob Agents. 2010;35(2):114-8.

15. Khoshnood S, Heidary M, Asadi A, Soleimani S, Motahar M, Savari M, Saki M, Abdi M. A review on mechanism of action, resistance, synergism, and clinical implications of mupirocin against Staphylococcus aureus. Biomed Pharmacother. 2019;109:1809-18.

16. Hetem DJ, Bonten MJ. Clinical relevance of mupirocin resistance in Staphylococcus aureus. J Hosp Infect. 2013;85(4):249-56.

17. Lee AS, Gizard Y, Empel J, Bonetti EJ, Harbarth S, François P. Mupirocininduced mutations in iles in various genetic backgrounds of methicillinresistant Staphylococcus aureus. J Clin Microbiol. 2014;52(10):3749-54.

18. Antonio M, McFerran N, Pallen MJ. Mutations affecting the Rossman fold of isoleucyl-tRNA synthetase are correlated with low-level mupirocin resistance in Staphylococcus aureus. Antimicrob Agents Chemother. 2002;46(2):438-42.

19. O'Neill AJ, McLaws F, Kahlmeter G, Henriksen AS, Chopra I. Genetic basis of resistance to fusidic acid in staphylococci. Antimicrob Agents Chemother. 2007;51(5):1737-40.

20. Liu Y, Geng W, Yang Y, Wang C, Zheng Y, Shang Y, Wu D, Li X, Wang L, Yu $S$, et al. Susceptibility to and resistance determinants of fusidic acid in Staphylococcus aureus isolated from Chinese children with skin and soft tissue infections. FEMS Immunol Med Microbiol. 2012;64(2):212-8.

21. Farrell DJ, Castanheira M, Chopra I. Characterization of global patterns and the genetics of fusidic acid resistance. Clin Infect Dis. 2011;52(Suppl 7):S487-92.

22. Nagaev I, Björkman J, Andersson DI, Hughes D. Biological cost and compensatory evolution in fusidic acid-resistant Staphylococcus aureus. Mol Microbiol. 2001:40(2):433-9.

23. Lannergård J, Norström T, Hughes D. Genetic determinants of resistance to fusidic acid among clinical bacteremia isolates of Staphylococcus aureus. Antimicrob Agents Chemother. 2009;53(5):2059-65.

24. Besier S, Ludwig A, Brade V, Wichelhaus TA. Molecular analysis of fusidic acid resistance in Staphylococcus aureus. Mol Microbiol. 2003;47(2):463-9.

25. Rijnders MI, Wolffs PF, Hopstaken RM, den Heyer M, Bruggeman CA, Stobberingh EE. Spread of the epidemic European fusidic acid-resistant impetigo clone (EEFIC) in general practice patients in the south of the Netherlands. J Antimicrob Chemother. 2012;67(5):1176-80.

26. Castanheira M, Watters AA, Bell JM, Turnidge JD, Jones RN. Fusidic acid resistance rates and prevalence of resistance mechanisms among Staphylococcus spp. isolated in North America and Australia, 2007-2008. Antimicrob Agents Chemother. 2010;54(9):3614-7.

27. Chen CM, Huang M, Chen HF, Ke SC, Li CR, Wang JH, Wu LT. Fusidic acid resistance among clinical isolates of methicillin-resistant Staphylococcus aureus in a Taiwanese hospital. BMC Microbiol. 2011;11:98.

28. Castanheira M, Watters AA, Mendes RE, Farrell DJ, Jones RN. Occurrence and molecular characterization of fusidic acid resistance mechanisms among Staphylococcus spp. from European countries (2008). J Antimicrob Chemother. 2010;65(7):1353-8.

29. Harrington AT, Black JA, Clarridge JE 3rd. In vitro activity of retapamulin and antimicrobial susceptibility patterns in a longitudinal collection of methicillin-resistant Staphylococcus aureus isolates from a veterans affairs medical center. Antimicrob Agents Chemother. 2015;60(3):1298-303.

30. McNeil JC, Hulten KG, Kaplan SL, Mason EO. Decreased susceptibilities to retapamulin, mupirocin, and chlorhexidine among Staphylococcus aureus isolates causing skin and soft tissue infections in otherwise healthy children. Antimicrob Agents Chemother. 2014;58(5):2878-83.

31. Saravolatz LD, Pawlak J, Saravolatz SN, Johnson LB. In vitro activity of retapamulin against Staphylococcus aureus resistant to various antimicrobial agents. Antimicrob Agents Chemother. 2013;57(9):4547-50.
32. Feßler AT, Wang Y, Wu C, Schwarz S. Mobile lincosamide resistance genes in staphylococci. Plasmid. 2018;99:22-31.

33. Takano T, Hung WC, Shibuya M, Higuchi W, Iwao Y, Nishiyama A, Reva I, Khokhlova OE, Yabe S, Ozaki K, et al. A new local variant (ST764) of the globally disseminated ST5 lineage of hospital-associated methicillin-resistant Staphylococcus aureus (MRSA) carrying the virulence determinants of community-associated MRSA. Antimicrob Agents Chemother. 2013;57(4): 1589-95.

34. Nakaminami $H$, Noguchi $N$, Ito A, Ikeda M, Utsumi K, Maruyama $H$, Sakamoto H, Senoo M, Takasato Y, Nishinarita S. Characterization of methicillin-resistant Staphylococcus aureus isolated from tertiary care hospitals in Tokyo. Japan J Infect Chemother. 2014;20(8):512-5.

35. He C, Xu S, Zhao H, Hu F, Xu X, Jin S, Yang H, Gong F, Liu Q. Leukotoxin and pyrogenic toxin superantigen gene backgrounds in bloodstream and wound Staphylococcus aureus isolates from eastern region of China. BMC Infect Dis. 2018;18(1):395

36. Yu F, Liu Y, Lv J, Qi X, Lu C, Ding Y, Li D, Liu H, Wang L. Antimicrobial susceptibility, virulence determinant carriage and molecular characteristics of Staphylococcus aureus isolates associated with skin and soft tissue infections. Braz J Infect Dis. 2015;19(6):614-22.

37. Traczewski MM, Brown SD. Proposed MIC and disk diffusion microbiological cutoffs and spectrum of activity of retapamulin, a novel topical antimicrobial agent. Antimicrob Agents Chemother. 2008;52(11):3863-7.

38. Yang JA, Park DW, Sohn JW, Yang IS, Kim KH, Kim MJ. Molecular analysis of isoleucyl-tRNA synthetase mutations in clinical isolates of methicillinresistant Staphylococcus aureus with low-level mupirocin resistance. J Korean Med Sci. 2006;21(5):827-32.

39. LaMarre J, Mendes RE, Szal T, Schwarz S, Jones RN, Mankin AS. The genetic environment of the $\mathrm{cfr}$ gene and the presence of other mechanisms account for the very high linezolid resistance of Staphylococcus epidermidis isolate 426-3147L. Antimicrob Agents Chemother. 2013;57(3):1173-9.

40. Haroche J, Allignet J, Buchrieser C, El Solh N. Characterization of a variant of $\mathrm{vga}(\mathrm{A})$ conferring resistance to streptogramin a and related compounds. Antimicrob Agents Chemother. 2000;44(9):2271-5.

41. Tsiodras S, Gold HS, Sakoulas G, Eliopoulos GM, Wennersten C, Venkataraman L, Moellering RC, Ferraro MJ. Linezolid resistance in a clinical isolate of Staphylococcus aureus. Lancet. 2001;358(9277):207-8.

42. McDougal LK, Steward CD, Killgore GE, Chaitram JM, MCAllister SK, Tenover FC. Pulsed-field gel electrophoresis typing of oxacillin-resistant Staphylococcus aureus isolates from the United States: establishing a national database. J Clin Microbiol. 2003;41(11):5113-20.

43. Enright MC, Day NP, Davies CE, Peacock SJ, Spratt BG. Multilocus sequence typing for characterization of methicillin-resistant and methicillin-susceptible clones of Staphylococcus aureus. J Clin Microbiol. 2000;38(3):1008-15.

\section{Publisher's Note}

Springer Nature remains neutral with regard to jurisdictional claims in published maps and institutional affiliations.

\section{Ready to submit your research? Choose BMC and benefit from:}

- fast, convenient online submission

- thorough peer review by experienced researchers in your field

- rapid publication on acceptance

- support for research data, including large and complex data types

- gold Open Access which fosters wider collaboration and increased citations

- maximum visibility for your research: over $100 \mathrm{M}$ website views per year

At $\mathrm{BMC}$, research is always in progress.

Learn more biomedcentral.com/submissions 